\title{
VISUAL CHARACTERIZATION OF ASSOCIATIVE QUASITRIVIAL NONDECREASING OPERATIONS ON FINITE CHAINS
}

\author{
GERGELY KISS
}

\begin{abstract}
In this paper we provide visual characterization of associative quasitrivial nondecreasing operations on finite chains. We also provide a characterization of bisymmetric quasitrivial nondecreasing binary operations on finite chains. Finally, we estimate the number of operations belonging to the previous classes.
\end{abstract}

\section{INTRODUCTION}

The study of aggregation operations defined on finite ordinal scales (i.e, finite chains) have been in the center of interest in the last decades, e.g., 6, 9, 14, 19, 2126, 28, 32,33. Among these operations, discrete uninorms has an important role in fuzzy logic and decision making [2, 4, 16.

In this paper we investigate associative quasitrivial nondecreasing operations on finite chains. In [7,10,29] idempotent discrete uninorms (i.e. idempotent symmetric nondecreasing associative operations with neutral elements defined on finite chains) have been characterized. Since every idempotent uninorm is quasitrivial (see e.g. [5]), in some sense this paper is a continuation of these works where we eliminate the assumption of symmetry of the operations.

Now we recall the analogue results for the unit interval $[0,1]$ as follows. CzogalaDrewniak proved in [5] that an associative monotonic idempotent operation with neutral element is a combination of minimum and maximum, and thus these are quasitrivial. Martin, Mayor and Torrens in 20, gave a complete characterization of associative quasitrivial nondecreasing operations on $[0,1]$. A refinement of their argument can be found in [30. (For the multivariable generalization of these results see [17.) We note that in [29] the analogue of the result of Czogała-Drewniak for finite chains has been provided assuming of symmetry of such operations.

The study of $n$-ary operations $F: X^{n} \rightarrow X$ satisfying the associativity property (see Definition 2.1) stemmed from the work of Dörnte [11] and Post [27]. In [12,13] the reducibility (see Definition 2.2) of associative $n$-ary operations have been studied by adjoining neutral elements. In [1] a complete characterization of quasitrivial associative $n$-ary operations have been presented. In 10 the quasitrivial symmetric nondecreasing associative $n$-ary operations defined on chains have been characterized. Recently, in [18] it was proved that associative idempotent nondecreasing $n$ ary operations defined on any chain are reducible. Using reducibility (see Theorem 3.1) a characterization of associative quasitrivial nondecreasing $n$-ary operations

Date: October 29, 2018.

2010 Mathematics Subject Classification. Primary 20N15, 39B72; Secondary 20M14.

Key words and phrases. associativity, bisymmetry, quasitriviality, characterization. 
for any $2 \leq n \in \mathbb{N}$ can be obtained automatically by a characterization of associative quasitrivial nondecreasing binary operations.

The paper is organized as follows. In Section 2 we present the most important definitions. In Section 3, we recall ( [18, Theorem 4.8]) the reducibility of associative idempotent nondecreasing $n$-ary operations and, hence, in the sequel we mainly focus on the binary case. We introduce the basic concept of visualization for quasitrivial monotone binary operations and present some preliminary results due to this concept. Here we discuss an important visual test of non-associativity (Lemma 3.5). Section 4 4 is devoted to the visual characterization of associative quasitrivial nondecreasing operations with so-called 'downward-right paths' (Theorems 4.12 and 4.13). We also present an Algorithm which provides the contour plot of any associative quasitrivial nondecreasing operation. In Section 5 we characterize the bisymmetric quasitrivial nondecreasing binary operations (Theorem [5.3). In Section 6 we calculate the number of associative quasitrivial nondecreasing operations defined on a finite chain of given size with and also without the assumption of the existence of neutral elements (Theorem 6.1). We get similar estimations for the number of bisymmetric quasitrivial nondecreasing binary operations defined on a finite chain of given size. (Proposition 6.5). In Section 7 we present some problems for further investigation. Finally, using a slight modification of the proof of [18, Theorem 3.2], in the Appendix we show that every associative quasitrivial monotonic $n$-ary operations are nondecreasing.

\section{Definition}

Here we present the basic definitions and some preliminary results. First we introduce the following simplification. For any integer $l \geq 0$ and any $x \in X$, we set $l \cdot x=x, \ldots, x$ (ltimes). For instance, we have $F\left(3 \cdot x_{1}, 2 \cdot x_{2}\right)=F\left(x_{1}, x_{1}, x_{1}, x_{2}, x_{2}\right)$.

Definition 2.1. Let $X$ be an arbitrary nonempty set. A operation $F: X^{n} \rightarrow X$ is called

- idempotent if $F(n \cdot x)=x$ for all $x \in X$;

- quasitrivial (or conservative) if

$$
F\left(x_{1}, \ldots, x_{n}\right) \in\left\{x_{1}, \ldots, x_{n}\right\}
$$

for all $x_{1}, \ldots, x_{n} \in X$;

- (n-ary) associative if

$$
\begin{aligned}
& F\left(x_{1}, \ldots, x_{i-1}, F\left(x_{i}, \ldots, x_{i+n-1}\right), x_{i+n}, \ldots, x_{2 n-1}\right) \\
& \quad=F\left(x_{1}, \ldots, x_{i}, F\left(x_{i+1}, \ldots, x_{i+n}\right), x_{i+n+1}, \ldots, x_{2 n-1}\right)
\end{aligned}
$$

for all $x_{1}, \ldots, x_{2 n-1} \in X$ and all $i \in\{1, \ldots, n-1\}$;

- (n-ary) bisymmetric if

$$
F\left(F\left(\mathbf{r}_{1}\right), \ldots, F\left(\mathbf{r}_{n}\right)\right)=F\left(F\left(\mathbf{c}_{1}\right), \ldots, F\left(\mathbf{c}_{n}\right)\right)
$$

for all $n \times n$ matrices $\left[\mathbf{r}_{1} \cdots \mathbf{r}_{n}\right]=\left[\begin{array}{lll}\mathbf{c}_{1} & \cdots & \mathbf{c}_{n}\end{array}\right]^{T} \in X^{n \times n}$.

We say that $F: X^{n} \rightarrow X$ has a neutral element $e \in X$ if for all $x \in X$ and all $i \in\{1, \ldots, n\}$

$$
F((i-1) \cdot e, x,(n-i) \cdot e)=x .
$$

Hereinafter we simply write that an $n$-ary operation is associative or bisymmetric if the context clarifies the number of its variables. We also note that if $n=2$ we 
VISUALIZATION OF ASSOCIATIVE QUASITRIVIAL NONDECREASING OPERATIONS 3

get the binary definition of associativity, quasitriviality, idempotency, and neutral element property.

Let $(X, \leq)$ be a nonempty chain (i.e, a totally ordered set). An operation $F: X^{n} \rightarrow$ $X$ is said to be

- nondecreasing (resp. nonincreasing) if

$$
F\left(x_{1}, \ldots, x_{n}\right) \leq F\left(x_{1}^{\prime}, \ldots, x_{n}^{\prime}\right) \quad\left(\text { resp. } F\left(x_{1}, \ldots, x_{n}\right) \geq F\left(x_{1}^{\prime}, \ldots, x_{n}^{\prime}\right)\right)
$$

whenever $x_{i} \leq x_{i}^{\prime}$ for all $i \in\{1, \ldots, n\}$,

- monotone in the $i$-th variable if for all fixed elements $a_{1}, \ldots a_{i-1}, a_{i+1}, \ldots, a_{n}$ of $X$, the 1-ary function defined as

$$
f_{i}(x):=F\left(a_{1}, \ldots, a_{i-1}, x, a_{i+1}, \ldots, a_{n}\right)
$$

is nondecreasing or nonincreasing.

- monotone if it is monotone in each of its variables.

Definition 2.2. We say that $F: X^{n} \rightarrow X$ is derived from a binary operation $G: X^{2} \rightarrow X$ if $F$ can be written of the form

$$
F\left(x_{1}, \ldots, x_{n}\right)=x_{1} \circ \cdots \circ x_{n},
$$

where $x \circ y=G(x, y)$. It is easy to see that $G$ is associative (and $F$ is $n$-ary associative) if and only if (1) is well-defined. If such a $G$ exists, then we say that $F$ is reducible.

We denote the diagonal of $X^{2}$ by $\Delta_{X}=\{(x, x): x \in X\}$.

Definition 2.3. Let $L_{k}$ denote $\{1, \ldots, k\}$ endowed with the natural ordering $(\leq)$.

Then $L_{k}$ is a finite chain. Moreover, every finite chain with $k$ element can be identified with $L_{k}$ and the domain of an $n$-variable operation defined on a finite chain can be identified with $\underbrace{L_{k} \times \cdots \times L_{k}}_{n}=\left(L_{k}\right)^{n}$ for some $k \in \mathbb{N}$.

For an arbitrary poset $(X, \leq)$ and $a \leq b \in X$ we denote the elements between $a$ and $b$ by $[a, b] \subseteq X$. In particular, for $L_{k}$

$$
[a, b]=\left\{m \in L_{k}: a \leq m \leq b\right\} .
$$

We also introduce the lattice notion of the minimum $(\wedge)$ and the maximum $(\vee)$ as follows

$$
\begin{aligned}
& x_{1} \wedge \cdots \wedge x_{n}=\wedge_{i=1}^{n} x_{i}=\min \left\{x_{1}, \ldots, x_{n}\right\}, \\
& x_{1} \vee \cdots \vee x_{n}=\vee_{i=1}^{n} x_{i}=\max \left\{x_{1}, \ldots, x_{n}\right\} .
\end{aligned}
$$

The binary operations $\operatorname{Proj}_{x}$ and $\operatorname{Proj}_{y}$ denote the projection to first and the second coordinate, respectively. Namely, $\operatorname{Proj}_{x}(x, y)=x$ and $\operatorname{Proj}_{y}(x, y)=y$ for all $x, y \in X$.

\section{BASIC CONCEPT AND PRELIMINARY RESULTS}

The following general result was published as [18, Theorem 4.8] recently.

Theorem 3.1. Let $X$ be a nonempty chain and $F: X^{n} \rightarrow X(n \geq 2)$ be an associative idempotent nondecreasing operation. Then there exists uniquely an associative idempotent nondecreasing binary operation $G: X^{2} \rightarrow X$ such that $F$ is derived from $G$. Moreover, $G$ can be defined by

$$
G(a, b)=F(a,(n-1) \cdot b)=F((n-1) \cdot a, b)(a, b \in X) .
$$


Remark 1. By the definition (2) of $G$, it is clear that if $F$ is quasitrivial, then $G$ is also.

According to Theorem 3.1 and Remark 1 a characterization of associative quasitrivial nondecreasing binary operations automatically implies a characterization for the $n$-ary case. Therefore, from now on we deal with the binary case $(n=2)$.

3.1. Visualization of binary operations. In this section we prove and reprove basic properties of quasitrivial associative nondecreasing binary operations in the spirit of visualization.

Observation 3.2. Let $X$ be a nonempty chain and let $F: X^{2} \rightarrow X$ be a quasitrivial monotone operation. If $F(x, t)=x$, then $F(x, s)=x$ for every $s \in[x \wedge t, x \vee t]$. Similarly, if $F(x, t)=t$, then $F(s, t)=t$ for every $s \in[x \wedge t, x \vee t]$.

A level-set of $F$ is a set of vertices of $L_{k}^{2}$ where $F$ has the same value. The contour plot of $F$ can be visualized by connecting the closest elements of the level-sets of $F$ by line segments. According to Observation 3.2, this contour plot can be drawn using only horizontal and vertical line segments starting from the diagonal (as in Figure 1.). It is clear that these lines do not cross each other by the monotonicity of $F$.

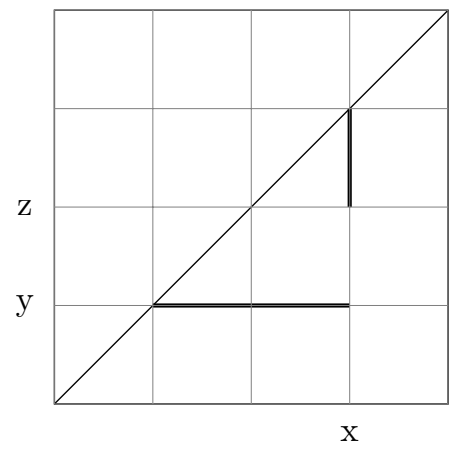

Figure 1. $F(x, y)=y$ and $F(x, z)=x$

As a consequence we get the following.

Corollary 3.3. Let $X$ be a nonempty chain and $F: X^{2} \rightarrow X$ be a quasitrivial operation.

$$
F \text { is monotone } \Longleftrightarrow F \text { is nondecreasing. }
$$

Proof. We only need to prove that every monotone quasitrivial operation is nondecreasing.

As an easy consequence of Observation 3.2 and the quasitriviality of $F$, we have $F(s, x) \leq F(t, x)$ and $F(x, t) \leq F(s, t)$ for any $x, s, t \in X$ that satisfies $s \in[x, t]$. This implies that $F$ is nondecreasing in the first variable. Similar argument shows the statement for the second variable.

Remark 2. The analogue of Corollary 3.3 holds whenever $n>2$. The proof is essentially the same as the proof of [18, Theorem 3.10]. Thus we present it in Appendix A. 
In the sequel we are dealing with associative, quasitrivial and nondecreasing operations.

There are several know forms of the following proposition. This type of results was first proved in [20. The form as stated here is [7, Proposition 18].

Proposition 3.4. Let $X$ be an arbitrary nonempty set and let $F: X^{2} \rightarrow X$ be a quasitrivial operation. Then the following assertions are equivalent.

(i) $F$ is not associative.

(ii) There exist pairwise distinct $x, y, z \in X$ such that $F(x, y), F(x, z), F(y, z)$ are pairwise distinct.

(iii) There exists a rectangle in $X^{2}$ such that one of the vertices is on $\Delta_{X}$ and the three remaining vertices are in $X^{2} \backslash \Delta_{X}$ and pairwise disconnected.

Now we present a visual version of the previous statement if $F$ is nondecreasing.

Lemma 3.5. Let $X$ be chain and $F: X^{2} \rightarrow X$ a quasitrivial, nondecreasing operation. Then $F$ is not associative if and only if there are pairwise distinct elements $x, y, z \in X$ that give one of the following pictures.

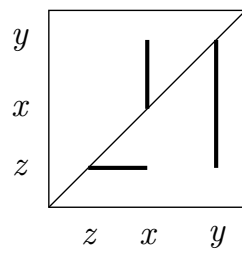

(a)

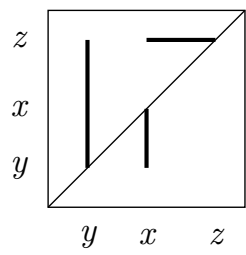

(b)

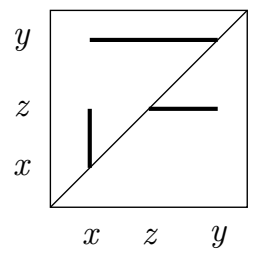

(c)

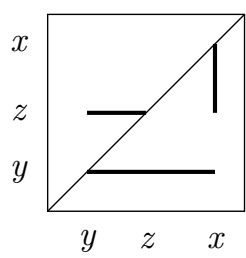

(d)

FiguRe 2. Four pictures that guarantee the non-associativity of $F$

Proof. By Proposition 3.4. $F$ is not associative if and only if there exists distinct $x, y, z \in X$ satisfying one of the following cases:

$$
F(x, y)=x, F(x, z)=z, F(y, z)=y(\text { Case } 1),
$$

or

$$
F(x, y)=y, F(y, z)=z, F(x, z)=x(\text { Case } 2) .
$$

Since $x, y, z \in X$ pairwise distinct elements, they can be ordered in 6 possible configuration of type $x<y<z$. For each case either (3) or (4) holds. Therefore we have 12 configurations as possible realizations of Case 1 or Case 2 .

Let us consider Case 1 (when equation (3) holds) and assume $x<y<z$. This implies the situation of Figure 3 .

The red point signs the problem of this configuration, since two lines with different values cross each other. There is no such a quasitrivial monotone operation.

Thus this subcase provides 'fake' example to study associativity. From the total, 8 cases are 'fake' in this sense.

The remaining 4 cases are presented in the statement. Figure 2 (a) and (b) represent the cases when equation (3) holds, and Figure2 (c) and (d) represent the cases when (4) holds. 


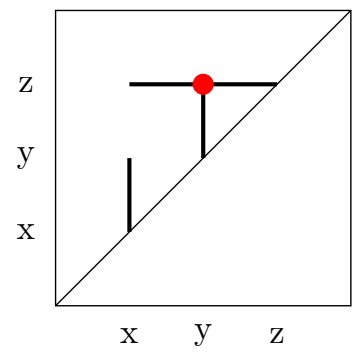

Figure 3. Case 1 and $x<y<z$ ('Fake' example)

Since for a 2-element set none of the cases of Figure 2 can be realized, as an immediate consequence of Lemma 3.5 we get the following.

Corollary 3.6. Every quasitrivial nondecreasing operation $F: L_{2}^{2} \rightarrow L_{2}$ is associative.

As a byproduct of this visualization we obtain a simple alternative proof for the following fact. This was proved first in [20, Proposition 2].

Corollary 3.7. Let $X$ be nonempty chain and $F: X^{2} \rightarrow X$ be a quasitrivial symmetric nondecreasing operation then $F$ is associative.

Proof. If we add the assumption of symmetry of $F$, each cases presented in Figure 2 have crossing lines (as in Figure 44), which is not possible. Thus $F$ is automatically associative.
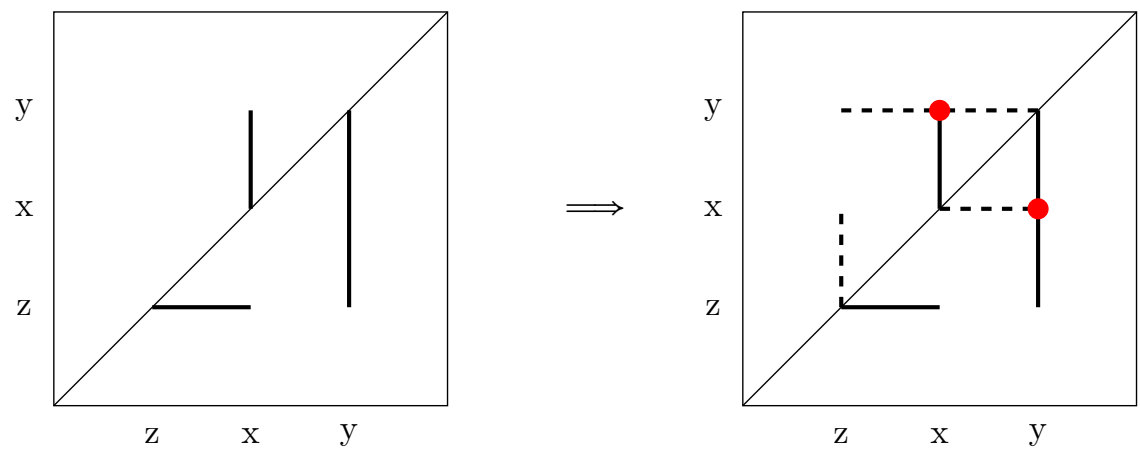

FIgURE 4. The symmetric case

For finite chains more can be stated.

Proposition 3.8 ( [7, Proposition 11.]). If $F: L_{k}^{2} \rightarrow L_{k}$ is quasitrivial symmetric nondecreasing then it is associative and has a neutral element.

Remark 3. The conclusion that $F$ has a neutral element is not necessarily true when $X=[0,1]$ (see [20]). This fact is one of the main difference between the cases $X=L_{k}$ and $X=[0,1]$. 
If we assume that $F$ has a neutral element (as it follows by Proposition 3.8 for finite chains), then as a consequence of Observation 3.2 we get the following pictures (Figure 5) for quasitrivial monotone operations having neutral elements. In Figure 5 the neutral element is denoted by $e$.
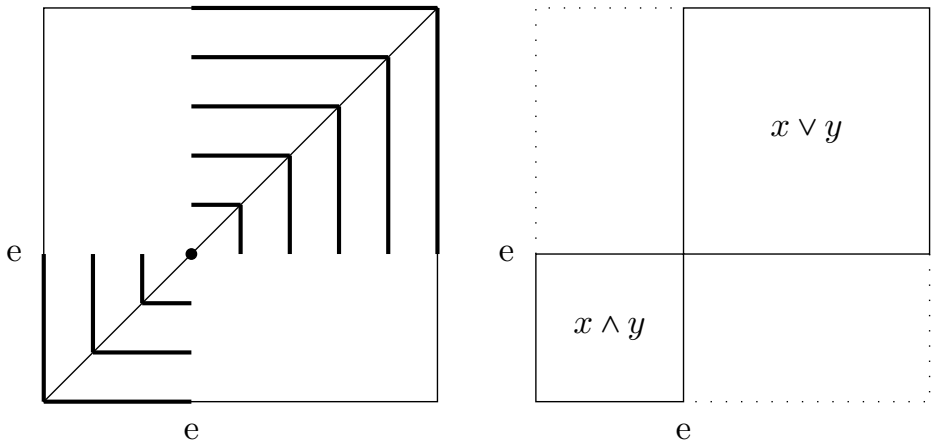

Figure 5. Partial description of a quasitrivial monotone operations having neutral elements

4. Visual CHARACTERIZATION OF ASSOCIATIVE QUASITRIVIAL NONDECREASING OPERATIONS DEFINED ON $L_{k}$

From now on we denote the upper and the lower 'triangle' by

$$
T_{1}=\left\{(x, y): x, y \in L_{k}, x \leq y\right\}, \quad T_{2}=\left\{(x, y): x, y \in L_{k}, x \geq y\right\},
$$

respectively, as in Figure 6. We note that $T_{1} \cap T_{2}$ is the diagonal $\Delta_{L_{k}}$.
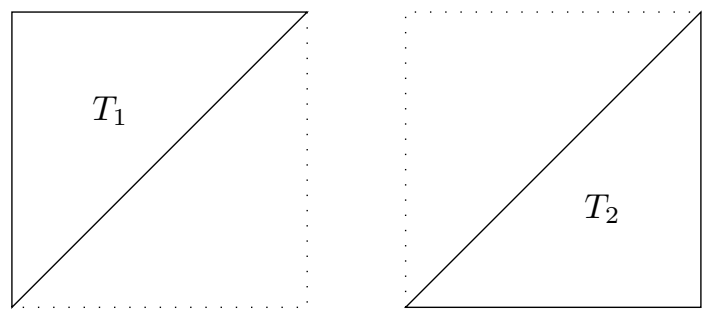

Figure 6 . The upper and lower 'triangles' $T_{1}$ and $T_{2}$

Definition 4.1. For a operation $F: L_{k}^{2} \rightarrow L_{k}$ there can be defined the upper symmetrization $F_{1}$ and lower symmetrization $F_{2}$ of $F$ as

$$
F_{1}(x, y)=\left\{\begin{array}{ll}
F(x, y) & \text { if }(x, y) \in T_{1} \\
F(y, x) & \text { if }(y, x) \in T_{1}
\end{array} \text { and } F_{2}(x, y)= \begin{cases}F(x, y) & \text { if }(x, y) \in T_{2} \\
F(y, x) & \text { if }(y, x) \in T_{2},\end{cases}\right.
$$

Briefly, $F_{1}(x, y)=F(x \wedge y, x \vee y), F_{2}(x, y)=F(x \vee y, x \wedge y) \quad \forall x, y \in L_{k}$.

Fodor [15] (see also [31, Theorem 2.6]) shown the following statement.

Proposition 4.2. Let $X$ be a nonempty chain and $F: X^{2} \rightarrow X$ be an associative operation. Then $F_{1}$ and $F_{2}$, the upper and the lower symmetrization of $F$, are also associative. 
This idea makes it possible to investigate the two 'parts' of a non-symmetric associative operation as one-one half of two symmetric associative operations.

By Proposition 3.8, both symmetrization of a nondecreasing quasitrivial operation $F: L_{k}^{2} \rightarrow L_{k}$ has a neutral element.

Definition 4.3. We call an element upper (or lower) half-neutral element of $F$ if it is the neutral element of the upper (or the lower) symmetrization. For simplicity we always denote the upper and lower half-neutral element of $F$ by $e$ and $f$, respectively.

Summarizing the previous results we get following partial description.

Proposition 4.4. Let $F: L_{k}^{2} \rightarrow L_{k}$ be an associative quasitrivial nondecreasing operation. Then it has an upper and an lower half-neutral element denoted by $e$ and $f$. Moreover, if $e \leq f$ then

$$
F(x, y)= \begin{cases}x \wedge y & \text { if } x \vee y \leq e \\ y & \text { if } e \leq x \leq f \\ x \vee y & \text { if } f \leq x \wedge y\end{cases}
$$

Analogously, if $f \leq e$ then

$$
F(x, y)= \begin{cases}x \wedge y & \text { if } x \vee y \leq f \\ x & \text { if } f \leq x \leq e \\ x \vee y & \text { if } e \leq x \wedge y\end{cases}
$$
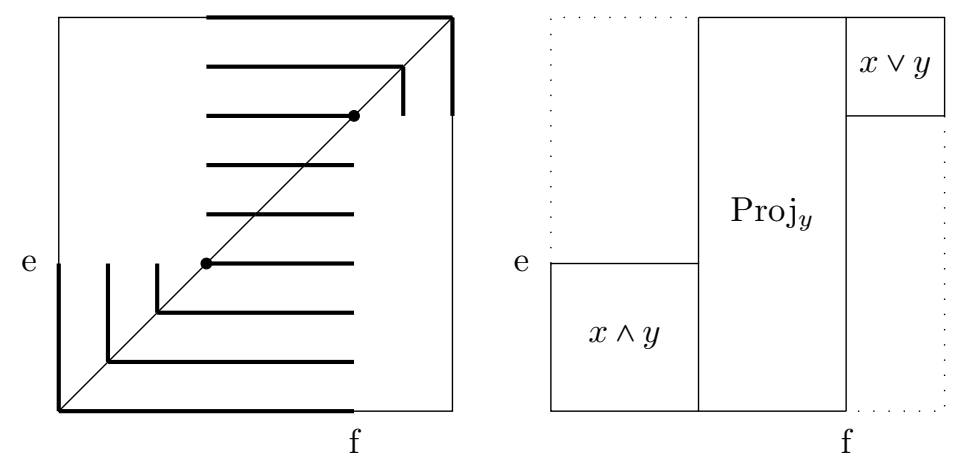

FiguRe 7. Partial description of associative quasitrivial monotone operations when $e \leq f$

We note that $e=f$ iff $F$ has a neutral element.

The following lemma is essential for the visual characterization.

Lemma 4.5. Let $F: L_{k}^{2} \rightarrow L_{k}$ be an associative quasitrivial nondecreasing operation. Assume that there exists $a<b \in L_{k}$ such that $F(a, b)=a$ and $F(b, a)=b$. Then one of the following holds:

(a) If $F(a+1, a)=a$, then

$$
F(x, b)=b \text { and } F(y, a)=a
$$

for every $x \in[a+1, b]$ and $y \in[a, b-1]$. 


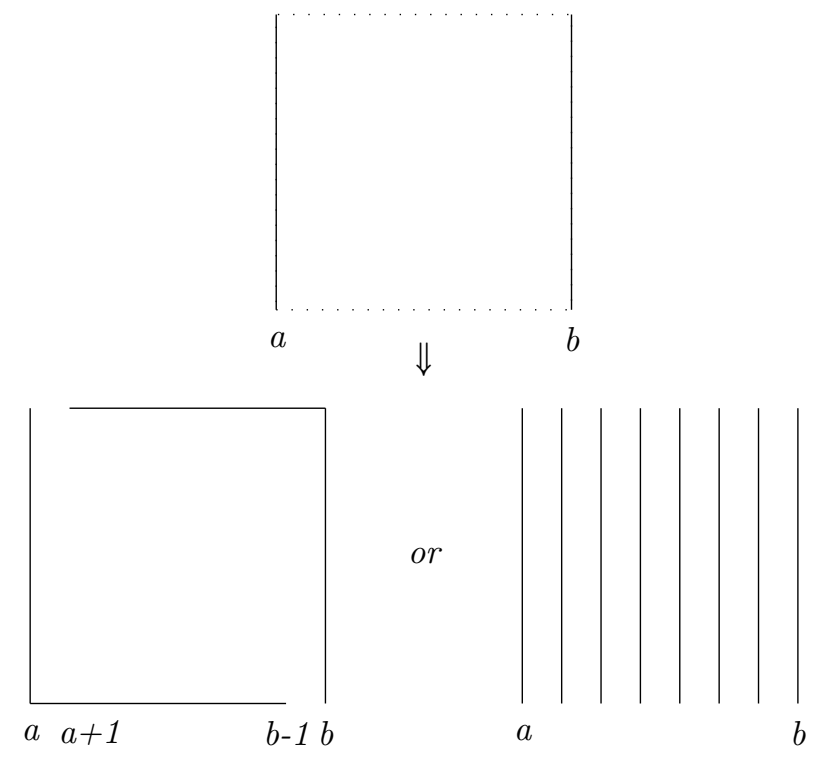

FIGURE 8. Graphical interpretation of Lemma 4.5

(b) If $F(a+1, a)=a+1$, then $F(x, y)=x\left(=\operatorname{Proj}_{x}\right)$ for all $x, y \in[a, b]$.

Proof. Assume first that $F(a+1, a)=a$. Then it follows that $F(a+1, b)=b$, otherwise we get Figure 2 (a). Using Observation 3.2 we have that $F(x, b)=b$ for every $x \in[a+1, b]$. The equation $F(b-1, b)=b$ implies that $F(b-1, a)=a$, otherwise we are in the situation of Figure 2 (b). Similarly, as above we get that $F(y, a)=a$ for every $y \in[a, b-1]$. Here we note that an analogue argument gives the same result if we assume originally that $F(b-1, b)=b$.

Now assume that $F(a+1, a)=a+1$. This immediately implies that $F(x, a)=x$ for every $x \in[a, b]$ by quasitriviality, since it cannot be $a$ by the nondecreasingness of $F$. Using Observation 3.2 again, it follows that $F(x, y)=x$ for all $y \in[a, x]$. Since $F(b-1, b)=b$ also implies the previous case, the assumption $F(a+1, a)=a+1$ implies $F(b-1, b)=b-1$. Similarly as above, this condition implies that $F(x, b)=x$ for all $x \in[a, b]$ and, by Observation [3.2, it follows that $F(x, y)=x$ for every $y \in[x, b]$. Altogether we get that $F(x, y)=x=\operatorname{Proj}_{x}(x, y)$ as we stated.

Remark 4. Analogue of Lemma 4.5 can be formalized as follows.

Let $F: L_{k}^{2} \rightarrow L_{k}$ be an associative quasitrivial nondecreasing operation. Assume that there exists $a<b \in L_{k}$ such that $F(b, a)=a$ and $F(a, b)=b$. Then one of the following holds:

(a) If $F(a, a+1)=a$, then

$$
F(b, x)=b \text { and } F(a, y)=a
$$

for every $x \in[a+1, b]$ and $y \in[a, b-1]$.

(b) If $F(a, a+1)=a+1$, then $F(x, y)=y\left(=\operatorname{Proj}_{y}\right)$ for all $x, y \in[a, b]$.

The proof of this statement is analogue to Lemma 4.5 using Figure 2(c) and (d) instead of Figure 2(a) and (b), respectively. 


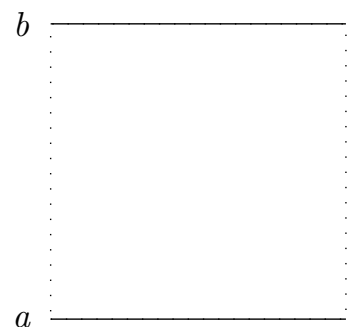

$\Downarrow$
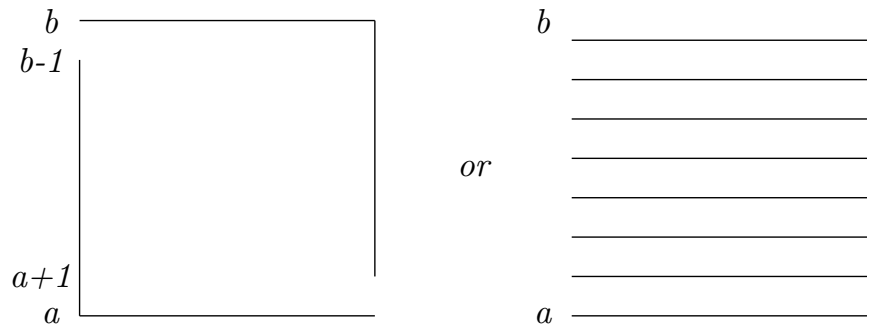

FiguRE 9. Graphical interpretation of Remark 4

From the previous results we conclude the following.

Lemma 4.6. Let $F: L_{k}^{2} \rightarrow L_{k}$ be an associative quasitrivial and nondecreasing operation and $e$ and $f$ the upper and the lower half-neutral elements, respectively, and let $a, b \in L_{k}(a<b)$ be given. If $F(x, y)=x$ for every $x, y \in[a, b]$ (i.e, Lemma 4.5 (b) holds), then $f<e$ and $[a, b] \subseteq[f, e]$. Similarly, if $F(x, y)=y$ for every $x, y \in[a, b]$ (i.e, Remark 4 (b) holds), then $e<f$ and $[a, b] \subseteq[e, f]$.

Proof. This is a direct consequence of Proposition 4.4. If $a$ or $b$ is not in $[e \wedge f, e \vee f]$ then $\tilde{F}=\left.F\right|_{[a, b]^{2}}$ contains a part where $\tilde{F}$ is a minimum or a maximum. Moreover, it is also easily follows that if $F(x, y)=x$ for every $x, y \in[a, b]$, then $f<e$ must hold. Similarly, $F(x, y)=y$ for every $x, y \in[a, b]$ implies $e<f$.

Corollary 4.7. Let $F, e, f$ be as in Lemma 4.6 and assume that $a, b \in X$ such that $a<b$ and $F(a, b) \neq F(b, a)$. Then

(i) Lemma 4.5(b) holds iff $f<e$ and $a, b \in[f, e]$,

(ii) Remark 4(b) holds iff $e<f$ and $a, b \in[e, f]$.

(iii) Lemma 4.5(a) or Remark \&(a) holds iff $a, b \notin[e \wedge f, e \vee f]$.

With other words we have:

Corollary 4.8. Let $F, e, f$ be as in Lemma 4.6. Then $F(a, b)=F(b, a)$, if $a \notin$ $[e \wedge f, e \vee f]$ and $b \in[e \wedge f, e \vee f]$, or $b \notin[e \wedge f, e \vee f]$ and $a \in[e \wedge f, e \vee f]$.

This form makes it possible to extend the partial description. (See Figure 10 for the case $e<f$.)

Using Lemma 4.5 and Remark 4 we can provide a visual characterization of associative quasitrivial nondecreasing operations. The characterization based on the following algorithm which outputs the contour plot of $F$. 

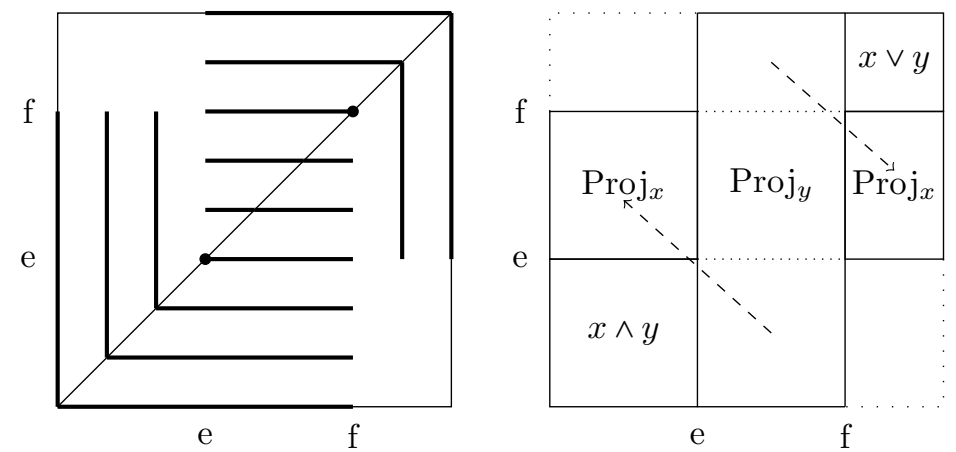

FIgURE 10. Extended partial description of associative quasitrivial monotone operations when $e<f$

Before we present the algorithm we note that the letters indicated in the following figures represent the value of operation $F$ in the corresponding points or lines (not a coordinate of the points itself as usual).

\section{Algorithm}

Initial setting: Let $Q_{1}=L_{k}^{2}$ and $F: L_{k}^{2} \rightarrow L_{k}$ be an associative quasitrivial nondecreasing operation.

Step i. For $Q_{i}=[a, b]^{2}(a \leq b)$ we distinguish cases according to the values of $F(a, b)$ and $F(b, a)$. Whenever $Q_{i}$ contains only 1 element $(a=b)$ for some $i$, then we are done.

I. (a) If $F(a, b)=F(b, a)=a$, then draw straight lines between the points $(b, a)$ and $(a, a)$ and between $(a, b)$ and $(a, a)$. Let $Q_{i+1}=[a+1, b]^{2}$. (See Figure [11)
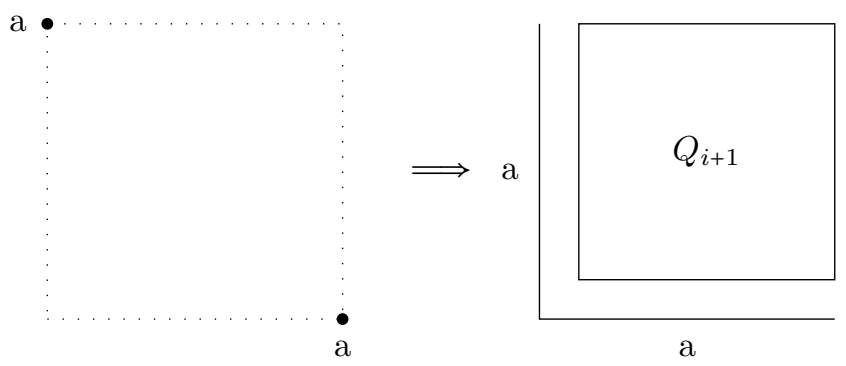

a

Figure 11. Case I.(a)

(b) If $F(a, b)=F(b, a)=b$, then draw straight lines between the points $(a, b)$ and $(b, b)$ and between $(b, a)$ and $(b, b)$. Let $Q_{i+1}=[a, b-1]^{2}$.

II. (a) If $F(a, b)=a, F(b, a)=b$ and $F(a+1, a)=a+1$, then $F(x, y)=x$ for all $x, y \in[a, b]$ and we are done. (See Figure 12)

(b) If $F(a, b)=b, F(b, a)=a$ and $F(a, a+1)=a+1$, then $F(x, y)=y$ for all $x, y \in[a, b]$ and we are also done.

III. (a) If $F(a, b)=a, F(b, a)=b$ and $F(a+1, a)=a$, then Lemma 4.5 (a) holds and we have Figure 13, Let $Q_{i+1}=[a+1, b-1]^{2}$. 


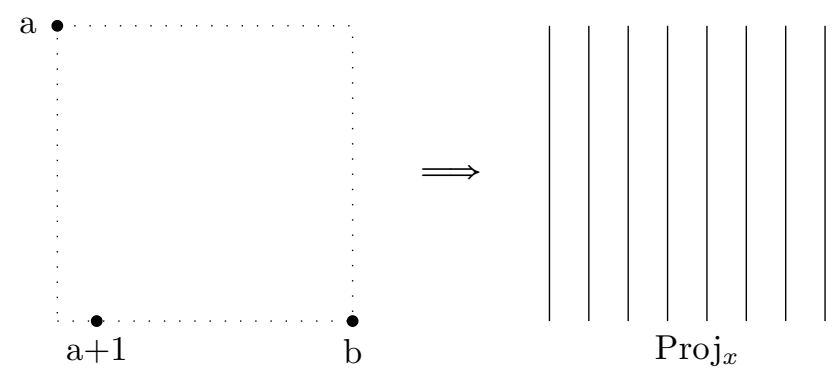

FiguRe 12. Case II.(a)

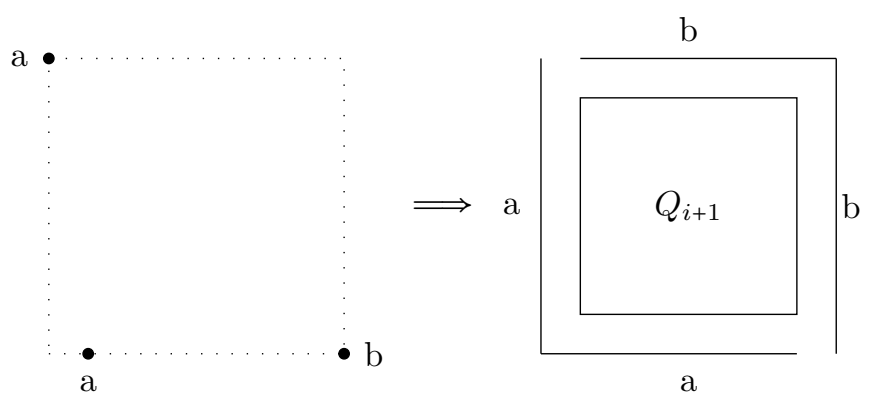

Figure 13. Case III.(a)

(b) If $F(a, b)=b, F(b, a)=a$ and $F(a, a+1)=a$, then Remark 4 (a) holds. Let $Q_{i+1}=[a+1, b-1]^{2}$.

It is clear that the algorithm is finished after finitely many steps. Let us denote this number of steps by $l \in \mathbb{N}$.

We also denote the top-left and the bottom-right corner of $Q_{i}$ by $p_{i}$ and $q_{i}$ $(i=1, \ldots, l)$, respectively.

Let $\mathcal{P}$ (and $\mathcal{Q}$ ) denote the path containing $p_{i}$ (and $q_{i}$ ) for $i \in\{1, \ldots, l\}$ and line segments between consecutive $p_{i}$ 's (and $q_{i}$ 's). Let us denote the line segment between $p_{i}$ and $p_{i+1}$ by $\overline{p_{i}, p_{i+1}}$. We set the notation $\mathcal{P}=\left(p_{j}\right)_{j=1}^{l}$ and $\mathcal{Q}=\left(q_{j}\right)_{j=1}^{l}$.

Clearly, we get the path $\mathcal{P}$ if we start at the top-left corner of $L_{k}^{2}$ and in each step we move either one place to the right or one place downward or one place diagonally downward-right.

Definition 4.9. We say that a path is a downward-right path of $L_{k}$ if in each step it moves to the nearest point of $L_{k}^{2}$ either one place to the right or one place downward or one place diagonally downward-right.

If $\overline{p_{i}, p_{i+1}}$ is horizontal or vertical, then the reduction from $Q_{i}$ to $Q_{i+1}$ is uniquely determined. Moreover, if $\overline{p_{i}, p_{i+1}}$ is horizontal, then $F(x, y)=F(y, x)=x \wedge y$, where $p_{i}=(x, y)$ and $q_{i}=(y, x)$. Similarly, if $\overline{p_{i}, p_{i+1}}$ is vertical, then $F(x, y)=F(y, x)=$ $x \vee y$, where $p_{i}=(x, y)$ and $q_{i}=(y, x)$. On the other hand if $\overline{p_{i}, p_{i+1}}$ is diagonal, then we have a free choice for the value of $F$ in $p_{i}$. This is determined by either Lemma 4.5 (a) or Remark 4 (a). Since in this case the value of $F$ in $q_{i}$ is different from $p_{i}$, the value in $q_{i}$ is automatically defined. It is also clear from the algorithm that the path $\mathcal{Q}$ is the reflection of $\mathcal{P}$ to the diagonal $\Delta_{L_{k}}$. 


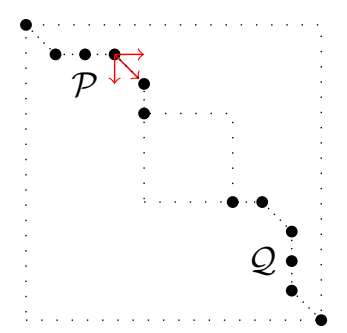

FiguRE 14. The path $\mathcal{P}$ is a downward-right path

Using the previous paragraph and Observation 3.2 it is possible to reconstruct operations from a given downward-right path $\mathcal{P}$ which starts at $p_{1}=(1, k)$.

Example 4.10. We illustrate the reconstruction on $L_{6} \times L_{6}$. The paths $\mathcal{P}=\left(p_{j}\right)_{j=1}^{5}$ and $\mathcal{Q}=\left(q_{j}\right)_{j=1}^{5}$ denoted by red and blue, respectively. According to the previous observations we get the following pictures (see Figure 15). It can be clearly seen that $\mathcal{Q}$ is the reflection of $\mathcal{P}$ to the diagonal $\Delta_{L_{6}}$, and 4 is the neutral element of the reconstructing operation, where $\mathcal{P}$ and $\mathcal{Q}$ touch each other and reach the diagonal $\Delta_{L_{6}}$. For the precise statement and proof see Theorem 4.13 .

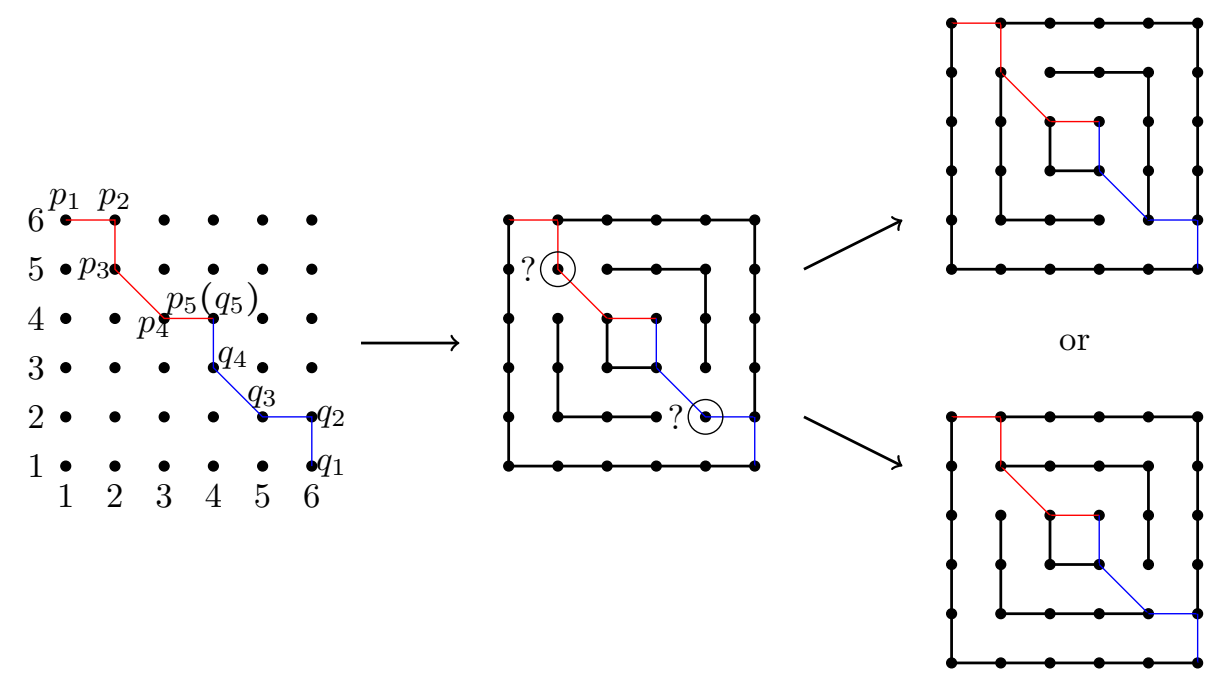

Figure 15. Reconstruction of $F$ from the path $\mathcal{P}$

Definition 4.11. Let $\mathcal{P} \subset L_{k}^{2}$ be the downward-right path from $(1, k)$ to $(a, b)$ $(a<b)$ and let $\mathcal{Q}$ be the reflection of $\mathcal{P}$ to the diagonal $\Delta_{L_{k}}$.

We say that $(x, y) \in L_{k}^{2} \backslash\left(\mathcal{P} \cup \mathcal{Q} \cup[a, b]^{2}\right)$ is above $\mathcal{P} \cup \mathcal{Q}$ if there exists $p=(x, w) \in \mathcal{P}$ such that $y>w$ or $q=(w, y) \in \mathcal{Q}$ such that $x>w$.

Similarly, we say that $(x, y) \in L_{k}^{2} \backslash\left(\mathcal{P} \cup \mathcal{Q} \cup[a, b]^{2}\right)$ is below $\mathcal{P} \cup \mathcal{Q}$ if there exists a $p=(x, w) \in \mathcal{P}$ such that $y<w$ or a $q=(w, y) \in \mathcal{Q}$ such that $x<w$.

Using this terminology we can summarize the previous observations and we get the following characterization. The next statement can be seen as the analogue of theorem of Czogała-Drewiak [5, Theorem 3.] for finite chains. 
Theorem 4.12. For every associative quasitrivial nondecreasing operation $F$ : $L_{k}^{2} \rightarrow L_{k}$ there exist half-neutral elements $a, b \in L_{k}(a \leq b)$ and a downward-right path $\mathcal{P}=\left(p_{j}\right)_{j=1}^{l}$ (for some $\left.l \in \mathbb{N}, l<k\right)$ from $(1, k)$ to $(a, b)$. We denote the reflection of $\mathcal{P}$ to the diagonal $\Delta_{L_{k}}$ by $\mathcal{Q}=\left(q_{j}\right)_{j=1}^{l}$. Then for every $(x, y) \notin \mathcal{P} \cup \mathcal{Q}$

$$
F(x, y)= \begin{cases}x \vee y, & \text { if }(x, y) \text { is above } \mathcal{P} \cup \mathcal{Q} \\ x \wedge y, & \text { if }(x, y) \text { is below } \mathcal{P} \cup \mathcal{Q} \\ \operatorname{Proj}_{x} \text { or } \operatorname{Proj}_{y}, & \text { if }(x, y) \in[a, b]^{2},\end{cases}
$$

and for every $(x, y) \in \mathcal{P} \cup \mathcal{Q}$

$$
F(x, y)= \begin{cases}x \wedge y & \text { if }(x, y)=p_{i} \text { or } q_{i} \text { and } \overline{p_{i}, p_{i+1}} \text { is horizontal, } \\ x \vee y, & \text { if }(x, y)=p_{i} \text { or } q_{i} \text { and } \overline{p_{i}, p_{i+1}} \text { is vertical, } \\ x \text { or } y, & \text { if }(x, y)=p_{i} \text { and } \overline{p_{i}, p_{i+1}} \text { is diagonal, } \\ x \text { or } y, & \text { if }(x, y)=q_{i} \text { and } \overline{q_{i}, q_{i+1}} \text { is diagonal. }\end{cases}
$$

If $a$ is the lower half-neutral element $f$ and $b$ is the upper half-neutral element $e$, then $F$ is $\operatorname{Proj}_{x}$ on $[a, b]^{2}$, otherwise it is $\operatorname{Proj}_{y}$.

Moreover $F$ is symmetric expect on $[a, b]^{2}$ and at the points $p_{i} \in \mathcal{P}$ and $q_{i} \in \mathcal{Q}$ where $\overline{p_{i}, p_{i+1}}$ is diagonal $(i \in\{1, \ldots, l-1\})$.

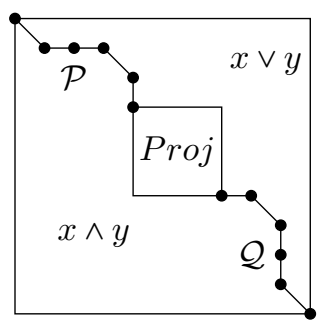

FiguRE 16. Characterization of associative quasitrivial nondecreasing operations on finite chains

Proof. The statement is clearly follows from the Algorithm and the definition of paths $\mathcal{P}$ and $\mathcal{Q}$.

The converse statement can be formalized as follows. This statement plays the role of theorem of Martin-Mayor-Torrens [20, Theorem 4.] for finite chains.

Theorem 4.13. Let $\mathcal{P}=\left(p_{j}\right)_{j=1}^{l}$ be a downward-right path in $T_{1} \subset L_{k}^{2}$ from $(1, k)$ to $(a, b)(a \leq b)$ and let $\mathcal{Q}=\left(q_{j}\right)_{j=1}^{l}$ be its reflection to the diagonal $\Delta_{L_{k}}$. Let $F: L_{k}^{2} \rightarrow L_{k}$ be defined for every $(x, y) \notin \mathcal{P} \cup \mathcal{Q}$ as

$$
F(x, y)= \begin{cases}x \vee y, & \text { if }(x, y) \text { is above } \mathcal{P} \cup \mathcal{Q}, \\ x \wedge y, & \text { if }(x, y) \text { is below } \mathcal{P} \cup \mathcal{Q}, \\ \text { Proj }_{x} \text { or } \operatorname{Proj}_{y} \text { (uniformly), } & \text { for every }(x, y) \in[a, b]^{2} .\end{cases}
$$

and for every $(x, y) \in \mathcal{P} \cup \mathcal{Q}$

$$
F(x, y)= \begin{cases}x \wedge y & \text { if }(x, y)=p_{i} \text { or } q_{i} \text { and } \overline{p_{i}, p_{i+1}} \text { is horizontal, } \\ x \vee y, & \text { if }(x, y)=p_{i} \text { or } q_{i} \text { and } \overline{p_{i}, p_{i+1}} \text { is vertical, } \\ x \text { or } y \text { (arbitrarily), }, & \text { if }(x, y)=p_{i} \text { and } \overline{p_{i}, p_{i+1}} \text { is diagonal. }\end{cases}
$$


If $(x, y)=q_{i}$ and $\overline{q_{i}, q_{i+1}}$ (or equivalently $\overline{p_{i}, p_{i+1}}$ ) is diagonal, then $F(x, y) \in\{x, y\}$ and $F(x, y) \neq F(y, x)$ uniquely define $F(x, y)$. Then $F$ is associative quasitrivial and nondecreasing.

Proof. It is clear that $F$ is defined for every $(x, y) \in L_{k}^{2}$ and $F$ is quasitrivial and nondecreasing. Now we show that $F$ is associative. If it is not the case, then by Lemma 3.5. one of the cases of Figure 2 is realized. Let $u, v, w \in L_{k}(u<v<w)$ denote the elements where its realized. Clearly $F(u, w) \neq F(w, u)$ and $F$ is not a projection on $[u, w]^{2}$. Thus, by the definition of $F$, it follows that $(u, w) \in \mathcal{P}$ and $(w, u) \in \mathcal{Q}$. Hence $p_{i}=(u, w)$ for some $i=\{1, \ldots, l-1\}$ and $\overline{p_{i}, p_{i+1}}$ is diagonal. Thus we have one of the following situation (Figure 17).
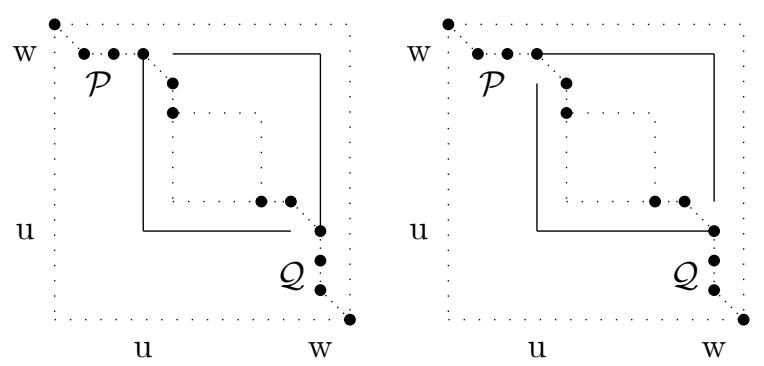

Figure 17. Two remaining cases

Therefore, since $u<v<w$, it follows that $F(u, v) \neq v, F(v, u) \neq v, F(w, v) \neq$ $v, F(v, w) \neq v$. Hence, none of the cases of Figure 2 can be realized. Thus $F$ is associative.

Remark 5. According to Theorems 4.12 and 4.13 it is clear that there is a surjection from the set of associative quasitrivial nondecreasing operations defined on $L_{k}$ to the downward-right paths defined on $T_{1}$ and started at $(1, k)$ (and ended somewhere in $T_{1}$ ). This surjection is a bijection if and only if the path $\mathcal{P}$ does not contain a diagonal move and $a=b$. This condition is equivalent that $F$ is symmetric (and has a neutral element).

Corollary 4.14. Let $F: L_{k}^{2} \rightarrow L_{k}$ be an associative quasitrivial nondecreasing operation. If $F$ is symmetric, then it is uniquely determined by a downward-right path $\mathcal{P}$ containing only horizontal and vertical line segments and it starts at $(1, k)$ and reaches the diagonal $\Delta_{L_{k}}$.

As a consequence of the previous corollary we obtain the result of [29, Theorem 4.] (see also [7, Theorem 14.]).

Corollary 4.15. The number of associative quasitrivial nondecreasing symmetric operation defined on $L_{k}$ is $2^{k-1}$.

Proof. Every path from $(1, k)$ to the diagonal $\Delta_{L_{k}}$ using right or downward moves contains $k$ points. According to Corollary 4.14 in each point of the path, except the last one, we have two options which direction we move further. This immediately implies that the number of associative quasitrivial nondecreasing symmetric operation defined on $L_{k}$ is $2^{k-1}$. 
In Theorem 6.1, as an application of the results of this section, we calculate the number of associative quasitrivial nondecreasing operations defined on $L_{k}$ and also the number of associative quasitrivial nondecreasing operations on $L_{k}$ that have neutral elements.

Remark 6. (a) We note that from the proof of Lemma 4.5 throughout this section we essentially use that $F$ is defined on a finite chain.

(b) In the continuous case [5,20 and also in the symmetric case 7,29 it is always possible to define a one variable function $g$, such that the extended graph of $g$ separates the points of the domain of the binary operation $F$ into two parts where $F$ is a minimum and a maximum, respectively. Now the paths $\mathcal{P}$ and $\mathcal{Q}$ play the role of the extended graph of $g$. Because of the diagonal moves of the path $\mathcal{P}$, it does not seems so clear how such a 'separating' function can be defined in the non-symmetric discrete case.

\section{BISYMMETRIC OPERATIONS}

In this section we show a characterization of bisymmetric quasitrivial nondecreasing binary operations based on the previous section. The following statement was proved as [7, Lemma 22.].

Lemma 5.1. Let $X$ be an arbitrary set and $F: X^{2} \rightarrow X$ be an operation. Then the following assertions hold.

(a) If $F$ is bisymmetric and has a neutral element, then it is associative and symmetric.

(b) If $F$ is bisymmetric and quasitrivial, then $F$ is associative.

(c) If $F$ is associative and symmetric, then it is bisymmetric.

Using also the results of Section 4 we get the following statement.

Theorem 5.2. Let $F: L_{k}^{2} \rightarrow L_{k}$ be a bisymmetric quasitrivial nondecreasing operation. Then there exists the upper half-neutral element $e$ and the lower half-neutral element $f$ and $F$ is symmetric on $\left(L_{k} \backslash[e \wedge f, e \vee f]\right)^{2}$.

Proof. According to Lemma 5.1(b), every quasitrivial bisymmetric operations are associative. Thus, by Proposition 4.4 it has an upper and lower half-neutral element ( $e$ and $f$, respectively).

Let us assume that $e \leq f$ (the case when $f \leq e$ can be handled similarly).

If there exists $u, v \in L_{k}$ such that $u<v, F(u, v) \neq F(v, u)$, then by Corollary 4.7. either $u, v \in[e, f]$ (then we do not need to prove anything) or $u, v \notin[e, f]$. Moreover, if $u, v \notin[e, f]$, then Lemma 4.5 (a) or Remark 4(a) holds. The existence of $e$ implies that $v-u \geq 2$.

If

$$
u=F(u, v) \neq F(v, u)=v
$$

is satisfied, then Lemma 4.5 (a) holds (i.e, $F(x, v)=v$ if $x \in[u+1, v]$ and $F(y, u)=u$ if $y \in[u, v-1])$. Since $v-u \geq 2, u+1 \leq v-1$, hence $F(u+1, u)=u$. On the other hand, $F$ is monotone and idempotent, thus by Observation $3.2, F(v, t)=v$ and $F(u, t)=u$ for all $t \in[u, v]$. Using bisymmetric equation we get the following $u=F(u, v)=F(F(u+1, u), F(v, v-1))=F(F(u+1, v), F(u, v-1))=F(v, u)=v$, which is a contradiction. 
Similarly, if

$$
v=F(u, v) \neq F(v, u)=u
$$

is satisfied, then Remark 4 (a) holds (i.e, $F(v, x)=v$ if $x \in[u+1, v]$ and $F(u, y)=u$ if $y \in[u, v-1])$. Since $v-u \geq 2, u+1 \leq v-1$, hence $F(v-1, v)=v$. Applying Observation 3.2 again, we have $F(t, v)=v$ and $F(t, u)=u$ for all $t \in[u, v]$. Using bisymmetric equation we get a contradiction as

$u=F(v, u)=F(F(v-1, v), F(u, u+1))=F(F(v-1, u), F(v, u+1))=F(u, v)=v$.

Applying Theorem 5.2 we get the following characterization.

Theorem 5.3. Let $F: L_{k}^{2} \rightarrow L_{k}$ be a quasitrivial nondecreasing operation. Then $F$ is bisymmetric if and only if there exists $a, b \in L_{k}(a \leq b)$ and a downward-right path $\mathcal{P}=\left(p_{j}\right)_{j=1}^{l}$ (for some $l \in \mathbb{N}$ ) from $(1, k)$ to $(a, b)$ containing only horizontal and vertical line segments such that for every $(x, y) \notin \mathcal{P} \cup \mathcal{Q}$

$$
F(x, y)= \begin{cases}x \vee y, & \text { if }(x, y) \text { is above } \mathcal{P} \cup \mathcal{Q}, \\ x \wedge y, & \text { if }(x, y) \text { is below } \mathcal{P} \cup \mathcal{Q}, \\ \text { Proj }_{x} \text { or } \text { Proj }_{y} \text { (uniformly), } & \text { for every }(x, y) \in[a, b]^{2} .\end{cases}
$$

and for every $(x, y) \in \mathcal{P} \cup \mathcal{Q}$

$$
F(x, y)= \begin{cases}x \wedge y & \text { if }(x, y)=p_{i} \text { or } q_{i} \text { and } \overline{p_{i}, p_{i+1}} \text { is horizontal, } \\ x \vee y, & \text { if }(x, y)=p_{i} \text { or } q_{i} \text { and } \overline{p_{i}, p_{i+1}} \text { is vertical, }\end{cases}
$$

where $\mathcal{Q}=\left(q_{j}\right)_{j=1}^{l}$ is the reflection of $\mathcal{P}$ to the diagonal $\Delta_{L_{k}}$.

In particular, $F$ is symmetric on $L_{k}^{2} \backslash[a, b]^{2}$ and one of the projections on $[a, b]^{2}$.

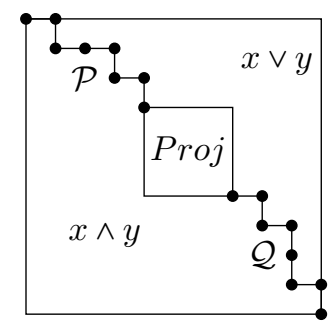

FiguRE 18. Characterization of bisymmetric quasitrivial nondecreasing operations on finite chains

Proof. (Necessity) Since $F$ is bisymmetric and quasitrivial, by Lemma 5.1 (b), $F$ is associative. By Theorem 4.12, there exist half-neutral elements $a, b \in L_{k}(a<b)$ and a downward-right path $\mathcal{P}$ from $(1, k)$ to $(a, b)$. By Theorem 5.3, $F$ is symmetric on $L_{k}^{2} \backslash[a, b]^{2}$. Thus $\mathcal{P}$ does not contain a diagonal line segment. Hence, applying again Theorem 4.12 we get that $F$ satisfies (5) and (6).

(Sufficiency) The operation $F$ defined by (5) and (6) satisfies the conditions of Theorem 4.13, thus $F$ is quasitrivial nondecreasing and associative. Now we show that $F$ is bisymmetric (i.e, $\forall u, v, w, z \in L_{k}$

$$
F(F(u, v), F(w, z))=F(F(u, w), F(v, z)) .)
$$


Let us assume that $F(x, y)=\operatorname{Proj}_{x}$ on $[a, b]^{2}$ (for $F(x, y)=\operatorname{Proj}_{y}$ on $[a, b]^{2}$ the proof is analogue). By Corollary 4.7, this implies that $a=f$ and $b=e(f<e)$ and, by Proposition 4.4 it is clear that

$$
F(x, y)=x \forall x \in L_{k}, \forall y \in[a, b] .
$$

Since $F$ is associative, we have

$$
F(F(u, v), F(w, z))=F(F(F(u, v), w), z)=F(F(u, F(v, w)), z)
$$

and

$$
F(F(u, w), F(v, z))=F(F(F(u, w), v), z)=F(F(u, F(w, v)), z) .
$$

If $F(v, w)=F(w, v)$, then (7) follows and we are done.

If $F(v, w) \neq F(w, v)$, then $v, w \in[a, b]^{2}$ and, since $F(x, y)=\operatorname{Proj}_{x}$ on $[a, b]^{2}$, $F(v, w)=v$ and $F(w, v)=w$. Then, by (8),

$$
\begin{aligned}
& F(F(u, F(v, w)), z)=F(F(u, v), z)=F(u, z), \\
& F(F(u, F(v, w)), z)=F(F(u, w), z)=F(u, z) .
\end{aligned}
$$

Thus $F$ is bisymmetric.

Remark 7. (a) There is a one-to-one correspondence between downward-right paths containing only vertical and horizontal line segments and the quasitrivial nondecreasing bisymmetric operations if we fix that the operation is $\operatorname{Proj}_{x}$ on $[a, b]^{2}$ ( $a$ and $b$ are the half neutral-elements of the operation). The same is true, if the operation is $\operatorname{Proj}_{y}$ on $[a, b]^{2}$.

(b) The nondecreasing assumption can be substituted by monotonicity. Indeed, by Corollary 3.3. monotonicity is equivalent with nondecreasingness for quasitrivial operations.

\section{The Number of OPERATIONS OF GIVEN ClASS}

This section is devoted to calculate the number of associative quasitrivial nondecreasing operations. Byproduct of the following argument we also consider the number of associative quasitrivial nondecreasing operations having neutral elements. With the same technique one can easily deduce the number of bisymmetric quasitrivial nondecreasing binary operations (see Proposition 6.5).

Theorem 6.1. Let $A_{k}$ denote the number of associative quasitrivial nondecreasing operations defined on $L_{k}$ and $B_{k}$ denote the number of associative quasitrivial nondecreasing operations defined on $L_{k}$ and having neutral elements. Then

$$
\begin{gathered}
A_{k}=\frac{1}{6}\left((2+\sqrt{3})(1+\sqrt{3})^{k}+(2-\sqrt{3})(1-\sqrt{3})^{k}-4\right), \\
B_{k}=\frac{1}{2 \cdot \sqrt{3}}\left((1+\sqrt{3})^{k}-(1-\sqrt{3})^{k}\right) .
\end{gathered}
$$

The following observations show that these numbers are related to the downwardright path $\mathcal{P}=\left(p_{j}\right)_{j=1}^{l}$ (for some $\left.l \leq k\right)$ in $T_{1}$ starting from $(1, k)$. Let $m_{\mathcal{P}}$ be the number of diagonal line segments $\overline{p_{i}, p_{i+1}} \in \mathcal{P}(i \in\{1, \ldots, l-1\})$. We say that the downward-right path $\mathcal{P}$ is weighted with weight $2^{m_{\mathcal{P}}}$.

Lemma 6.2. $\quad$ (a) $B_{k}$ is the sum of the weights of weighted paths that starts at $(1, k)$ and reaches $\Delta_{L_{k}}$. 
(b) $A_{k}+B_{k}$ is twice the sum of the weights of weighted paths in $T_{1}$ that starts at $(1, k)$ and ends at any point of $T_{1}$.

Proof. (a) Applying Theorem 4.12, it is clear that if an associative quasitrivial nondecreasing binary operation $F$ has a neutral element, then the downwardright path $\mathcal{P}$ defined for $F$ reaches the diagonal $\Delta_{L_{k}}$. By Theorem 4.13 , there can be defined $2^{m_{\mathcal{P}}}$ different operations for a given path $\mathcal{P}$ that reaches the diagonal, since we have a choice in each case when the path contains a diagonal line segment. This show the first part of the statement.

(b) This statement follows from the fact that for any associative quasitrivial nondecreasing operation $F$ one can define a downward-right path which starts at $(1, k)$ and ends somewhere in $T_{1}$. If its end in $(a, b)$ where $a<b$ (not on $\Delta_{L_{k}}$ ), then $F$ is one of the projections in $[a, b]^{2}$, and $a$ and $b$ are the half-neutral elements of $F$. This makes the extra 2 factor in the statement.

Let $\Pi_{1}$ denote set of the weighted paths in $T_{1}$ that starts at $(1, k)$ and ends at $(a, b)$ where $a<b$. Similary, $\Pi_{2}$ denote the set of weighted paths that starts at $(1, k)$ and reaches $\Delta_{L_{k}}$. Hence,

$$
A_{k}=2 \cdot \sum_{\mathcal{P} \in \Pi_{1}} 2^{m_{\mathcal{P}}}+\sum_{\mathcal{P} \in \Pi_{2}} 2^{m_{\mathcal{P}}}
$$

According to the (a) part

$$
B_{k}=\sum_{\mathcal{P} \in \Pi_{2}} 2^{m_{\mathcal{P}}} .
$$

Adding these equations, we get the statement for $A_{k}+B_{k}$.

Now we present a recursive formula for $A_{k}$ and $B_{k}$.

Lemma 6.3. (a) $B_{1}=1, B_{2}=2$ and $B_{k}=2 \cdot B_{k-1}+2 \cdot B_{k-2}$ for every $k \geq 3$.

(b) $A_{k}=2 \sum_{i=1}^{k} B_{i}-B_{k}$ for every $k \in \mathbb{N}$.

Proof. (a) $B_{1}=1, B_{2}=2$ are clear. The recursive formula follows from the Algorithm presented in Section 4 and the definition of downward-right path $\mathcal{P}=\left(p_{j}\right)_{j=1}^{l}$. Now we assume that $k \geq 3$. If $\overline{p_{1}, p_{2}}$ is horizontal or vertical, then Case I. (a) or (b) of the Algorithm holds (see also Figure 11). Thus we reduce the square $Q_{1}$ of size $k$ to a square $Q_{2}$ of size $k-1$. If $\overline{p_{1}, p_{2}}$ is diagonal, then Case III (a) or (b) holds (see also Figure13). Thus we reduce the square $Q_{1}$ of size $k$ to a square $Q_{2}$ of size $k-2$. By definition, the number of associative quasitrivial nondecreasing operations having neutral elements defined on a square of size $k$ is $B_{k}$. Thus we get that $B_{k}=2 \cdot B_{k-1}+2 \cdot B_{k-2}$.

(b) This follows from Lemma 6.2 (b) and the fact that 'sum of the weights of weighted paths from $(1, k)$ to any point of $T_{1}$ ' is exactly $\sum_{i=1}^{k} B_{i}$. Indeed, let $s \in\{1, \ldots, k\}$ be fixed. Then $B_{s}$ is equal to the sum of the weights of weighted paths $\mathcal{P}$ that starts at $(1, k)$ and ends at $(a, b)$ where $b-a=s$.

Proof of Theorem 6.1. We use a standard method of second-order linear recurrence equations for the formula of Lemma 6.3 (a). Therefore,

$$
B_{k}=c_{1} \cdot\left(\alpha_{1}\right)^{k}+c_{2}\left(\alpha_{2}\right)^{k}
$$


where $\alpha_{1}, \alpha_{2}\left(\alpha_{1}<\alpha_{2}\right)$ are the solutions of the equation $x^{2}-2 x-2=0$. Thus, $\alpha_{1}=1-\sqrt{3}, \alpha_{2}=1+\sqrt{3}$. By the initial condition $B_{1}=1$ and $B_{2}=2$, we get that $c_{1}=-c_{2}=\frac{1}{2 \sqrt{3}}$. Thus,

$$
B_{k}=\frac{1}{2 \cdot \sqrt{3}}\left((1+\sqrt{3})^{k}-(1-\sqrt{3})^{k}\right) .
$$

According to Lemma $6.3(\mathrm{~b}), A_{k}$ can be calculated as $2 \cdot \sum_{i=1}^{k} B_{i}-B_{k}$.

This provides that

$$
A_{k}=\frac{1}{6}\left((2+\sqrt{3})(1+\sqrt{3})^{k}+(2-\sqrt{3})(1-\sqrt{3})^{k}-4\right)
$$

Here we present a list of the first 10 value of $A_{k}: A_{1}=1, A_{2}=4, A_{3}=12, A_{4}=$ $34, A_{5}=94, A_{6}=258, A_{7}=706, A_{8}=1930, A_{9}=5274, A_{10}=14410$.

By Theorem 3.1, we get the similar results for the $n$-ary case.

Corollary 6.4. (a) The number of associative quasitrivial nondecreasing operations $F: L_{k}^{n} \rightarrow L_{k}(k \in \mathbb{N})$ having neutral elements is

$$
\frac{1}{2 \cdot \sqrt{3}}\left((1+\sqrt{3})^{k}-(1-\sqrt{3})^{k}\right),
$$

(b) The number of associative quasitrivial nondecreasing operations $F: L_{k}^{n} \rightarrow$ $L_{k}(k \in \mathbb{N})$ is

$$
\frac{1}{6}\left((2+\sqrt{3})(1+\sqrt{3})^{k}+(2-\sqrt{3})(1-\sqrt{3})^{k}-4\right) .
$$

Proposition 6.5. Let $C_{k}$ denote number of bisymmetric quasitrivial nondecreasing binary operations defined in $L_{k}$ and $D_{k}$ denote the number of bisymmetric quasitrivial nondecreasing binary operations having neutral elements. Then

$$
\begin{gathered}
D_{k}=2^{k-1}, \\
C_{k}=3 \cdot 2^{k-1}-2 .
\end{gathered}
$$

Proof. (a) By Lemma 5.1 and Proposition 3.8, bisymmetric quasitrivial nondecreasing binary operations having neutral elements defined on $L_{k}$ are exactly the associative quasitrivial symmetric nondecreasing binary operations. Thus by Corollary 4.15, we get that $D_{k}=2^{k-1}$.

(b) Same argument as in Lemma 6.3 (b) shows that $C_{k}=2 \sum_{i=1}^{k} D_{i}-D_{k}$. Using this we get that $C_{k}=2 \cdot\left(2^{k}-1\right)-2^{k-1}=3 \cdot 2^{k-1}-2$.

Remark 8. During the finalization of this paper the author have been informed that Miguel Couceiro, Jimmy Devillet and Jean-Luc Marichal found an alternative and independent approach for similar estimations in their upcoming paper [8].

\section{Open PROBLEMS AND FURTHER PERSPECTIVES}

First we summarize the most important results of our paper. In this article we introduced a geometric interpretation of quasitrivial nondecreasing associative binary operations. We gave a characterization of such operations on finite chains using downward-right paths. Combining this with a reducibility argument we provided characterization for the $n$-ary analogue of the problem. As a remarkable application of our visualization method we gave characterization of bisymmetric 
quasitrivial nondecreasing binary operation on finite chains. As a byproduct of our argument we estimated the number of operations belonging to these classes.

These results initiate the following open problems.

(1) Characterize the $n$-ary bisymmetric quasitrivial nondecreasing operations. If these operations are also associative, then we can apply reducibility to deduce a characterization for them. On the other hand if $n \geq 3$, then not all of such operations are associative as the following example shows. Let $F: X^{n} \rightarrow X(n \geq 3)$ be the projection on the $i^{t h}$ coordinate where $i$ is neither 1 or $n$. Then it is easy to show that it is bisymmetric quasitrivial nondecreasing but not associative.

(2) Find a visual characterization of associative idempotent nondecreasing operations. Quasitrivial operations are automatically idempotent. Since idempotent operations are essentially important in fuzzy logic, this problem has its own interest.

\section{ACKNOWLEDGEMENTS}

The author would like to thank Jimmy Devillet and the anonymous referee for the example given in the first open problem in Section 7. This research is supported by the internal research project R-AGR-0500 of the University of Luxembourg. The author was partially supported by the Hungarian Scientific Research Fund (OTKA) K104178.

\section{APPENDIX}

This section is devoted to prove the analogue of Corollary 3.3. As it was already mentioned in Remark 2 the proof is just a slight modification of the proof of [18, Theorem 3.2]. The difference is based on the following easy lemma.

Lemma 7.1. Let $X$ be a chain and $F: X^{n} \rightarrow X$ be an associative monotone operation. Then $F$ is non-decreasing in the first and the last variable.

Proof. The argument for the first and for the last variable is similar. We just consider it for the first variable. From the definition of associativity it is clear that an associative operation $F: X^{n} \rightarrow X$ is satisfies

$$
\begin{aligned}
& F\left(F\left(x_{1}, \ldots, x_{n}\right), x_{n+1}, \ldots, x_{2 n-1}\right)= \\
& F\left(x_{1}, F\left(x_{2}, \ldots, x_{n+1}\right), x_{n+2}, \ldots, x_{2 n-1}\right) .
\end{aligned}
$$

for every $x_{1}, \ldots, x_{2 n-1} \in X$. Now let us fix $x_{2}, \ldots, x_{2 n-1} \in X$ and define

$$
h(x)=F\left(F\left(x, x_{2}, \ldots, x_{n}\right), x_{n+1}, \ldots, x_{2 n-1}\right) .
$$

The operation $F$ is monotonic in the first variable thus it is clear that $h(x)$ is nondecreasing, since we apply $F$ twice when $x$ is in the first variable. Then using (9) we get that $F$ must be nondecreasing in the first variable.

As it was also mentioned in [18 the following condition is an easy application of [1, Theorem 1.4] using the statement therein for $A_{2}=\varnothing$.

Theorem 7.2. Let $X$ be an arbitrary set. Suppose $F: X^{n} \rightarrow X$ be a quasitrivial associative operation. If $F$ is not derived from a binary operation $G$, then $n$ is odd and there exist $b_{1}, b_{2}\left(b_{1} \neq b_{2}\right)$ such that for any $a_{1}, \ldots, a_{n} \in\left\{b_{1}, b_{2}\right\}$

$$
F\left(a_{1}, \ldots, a_{n}\right)=b_{i}(i=\{1,2\}),
$$


where $b_{i}$ occurs odd number of times.

Proposition 7.3. Let $X$ be a totally ordered set and let $F: X^{n} \rightarrow X$ be an associative, quasitrivial, monotone operation. Then $F$ is reducible.

Proof. According to Theorem 7.2 , if $F$ is not reducible, then $n$ is odd. Hence $n \geq 3$ and there exist $b_{1}, b_{2}$ satisfying equation (10). Since $b_{1} \neq b_{2}$, we may assume that $b_{1}<b_{2}$ (the case $b_{2}<b_{1}$ can be handled similarly). By the assumption (10) for $b_{1}$ and $b_{2}$ we have

$$
F\left(n \cdot b_{1}\right)=b_{1}, F\left(b_{2},(n-1) \cdot b_{1}\right)=b_{2}, F\left(b_{2},(n-2) \cdot b_{1}, b_{2}\right)=b_{1} .
$$

By Lemma 7.1, $F$ is nondecreasing in the first and the last variable. Thus we have

$$
F\left(n \cdot b_{1}\right) \leq F\left(b_{2},(n-1) \cdot b_{1}\right) \leq F\left(b_{2},(n-2) \cdot b_{1}, b_{2}\right) .
$$

This implies $b_{1}=b_{2}$, a contradiction.

The following was proved as [18, Corollary 4.9].

Corollary 7.4. Let $X$ be a nonempty chain and $n \geq 2$ be an integer. An associative, idempotent, monotone operation $F: X^{n} \rightarrow X$ is reducible if and only if $F$ is nondecreasing.

Using Proposition 7.3 and Corollary 7.4 we get the statement.

Corollary 7.5. Let $n \geq 2 \in \mathbb{N}$ be given, $X$ be a nonempty chain and $F: X^{n} \rightarrow X$ be an associative quasitrivial operation.

$$
F \text { is monotone } \Longleftrightarrow F \text { is nondecreasing. }
$$

\section{REFERENCES}

[1] N. L. Ackerman. A characterization of quasitrivial $n$-semigroups, to appear in Algebra Universalis.

[2] S. Berg and T. Perlinger. Single-peaked compatible preference profiles: some combinatorial results. Social Choice and Welfare 27(1), pp. 89-102, 2006.

[3] D. Black. On the rationale of group decision-making. J Polit Economy, 56(1), pp. 23-34, 1948.

[4] D. Black. The theory of committees and elections. Kluwer Academic Publishers, Dordrecht, 1987.

[5] E. Czogała and J. Drewniak. Associative monotonic operations in fuzzy set theory. Fuzzy Sets and Systems, 12(3), pp. 249-269, 1984.

[6] M. Couceiro and J.-L. Marichal. Representations and characterizations of polynomial operations on chains. J. of Mult.-Valued Logic \& Soft Computing, 16, pp. 65-86, 2010.

[7] M. Couceiro, J. Devillet, and J.-L. Marichal. Characterizations of idempotent discrete uninorms. Fuzzy Sets and Systems 334 (2018), 60-72.

[8] M. Couceiro, J. Devillet and J-L. Marichal, Quasitrivial semigroups: characterizations and enumerations. Preprint. https://arxiv.org/pdf/1709.09162.pdf

[9] B. De Baets and R. Mesiar. Discrete triangular norms. in Topological and Algebraic Structures in Fuzzy Sets, A Handbook of Recent Developments in the Mathematics of Fuzzy Sets, Trends in Logic, eds. S. Rodabaugh and E. P. Klement (Kluwer Academic Publishers), 20, pp. 389400, 2003.

[10] J. Devillet, G. Kiss and J.-L. Marichal. Characterizations of quasitrivial symmetric nondecreasing associative operations, submitted to Semigroup Forum.

[11] W. Dörnte. Untersuchengen über einen verallgemeinerten Gruppenbegriff. Math. Z. 29, pp. 1-19, 1928.

[12] W. A. Dudek and V. V. Mukhin. On topological n-ary semigroups. Quasigroups and Related Systems, 3, pp. 373-88, 1996. 
[13] W. A. Dudek and V. V. Mukhin. On n-ary semigroups with adjoint neutral element. Quasigroups and Related Systems, 14, pp. 163-168, 2006.

[14] J. Fodor. Smooth associative operations on finite ordinal scales. IEEE Trans. Fuzzy Systems, 8, pp. 791-795, 2000.

[15] J. Fodor An extension of Fung-Fu's theorem. Int. J. of Uncertainty, Fuzziness and Knowledge-Based Systems, 4(3), pp. 235-243, 1996.

[16] E. Foundas. Some results of Black's permutations. Journal or Discrete Mathematical Sciences and Cryptography, 4(1), pp. 47-55, 2001.

[17] G. Kiss and G. Somlai. A characterization of n-associative, monotone, idempotent functions on an interval that have neutral elements, Semigroup Forum, 96(3), pp. 438-451, 2018.

[18] G. Kiss and G. Somlai. Associative idempotent nondecreasing functions are reducible, accepted at Semigroup Forum, https://arxiv.org/pdf/1707.04341.pdf.

[19] G. Li, H.-W. Liu and J. Fodor. On weakly smooth uninorms on finite chain. Int. J. Intelligent Systems, 30, pp. 421-440, 2015.

[20] J. Martín, G. Mayor and J. Torrens. On locally internal monotonic operations. Fuzzy Sets and Systems, 137(1), pp. 27-42, 2003.

[21] M. Mas, G. Mayor and J. Torrens. t-operators and uninorms on a finite totally ordered set. Int. J. Intelligent Systems, 14, pp. 909-922, 1999.

[22] M. Mas, M. Monserrat and J. Torrens. On bisymmetric operators on a finite chain. IEEE Trans. Fuzzy Systems, 11, pp. 647-651, 2003.

[23] M. Mas, M. Monserrat and J. Torrens. On left and right uninorms on a finite chain. Fuzzy Sets and Systems, 146, pp. 3-17, 2004.

[24] M. Mas, M. Monserrat and J. Torrens. Smooth t-subnorms on finite scales. Fuzzy Sets and Systems, 167, pp. 82-91, 2011.

[25] G. Mayor and J. Torrens. Triangular norms in discrete settings. in Logical, Algebraic, Analytic, and Probabilistic Aspects of Triangular Norms, eds. E. P. Klement and R. Mesiar (Elsevier, Amsterdam), pp. 189-230, 2005.

[26] G. Mayor, J. Su ner and J. Torrens. Copula-like operations on finite settings. IEEE Trans. Fuzzy Systems, 13, pp. 468-477, 2005.

[27] E. L. Post. Polyadic groups, Trans. Amer. Math. Soc., 48, pp. 208-350, 1940.

[28] D. Ruiz-Aguilera and J. Torrens. A characterization of discrete uninorms having smooth underlying operators. Fuzzy Sets and Systems, 268, pp. 44-58, 2015.

[29] D. Ruiz-Aguilera, J. Torrens, B. De Baets and J. Fodor. Idempotent uninorms on finite ordinal scales. Int. J. of Uncertainty, Fuzziness and Knowledge-Based Systems 17 (1), pp. $1-14,2009$.

[30] D. Ruiz-Aguilera, J. Torrens, B. De Baets and J. Fodor. Some remarks on the characterization of idempotent uninorms. in: E. Hüllermeier, R. Kruse, F. Hoffmann (Eds.), Computational Intelligence for Knowledge-Based Systems Design, Proc. 13th IPMU 2010 Conference, LNAI, vol. 6178, Springer-Verlag, Berlin, Heidelberg, 2010, pp. 425-434.

[31] W. Sander Associative aggregation operators. In:Aggregation operators. New trends and applications, pp. 124-158. Stud. Fuzziness Soft Comput. Vol. 97. Physica-Verlag, Heidelberg, Germany, 2002.

[32] Y. Su and H.-W. Liu. Discrete aggregation operators with annihilator. Fuzzy Sets and Systems, 308, pp. 72-84, 2017.

[33] Y. Su, H.-W. Liu, and W. Pedrycz. On the discrete bisymmetry. IEEE Trans. Fuzzy Systems. To appear. DOI:10.1109/TFUZZ.2016.2637376

Mathematics Research Unit, University of Luxembourg, Maison du Nombre, 6 , avenue DE LA Fonte, L-4364 Esch-Sur-Alzette, Luxembourg

E-mail address: gergely.kiss[at]uni.lu 